\title{
2-Calabi-Yau tilted 代数的交换关系
}

\author{
刘品 \\ 西南交通大学数学学院, 成都 610031 \\ E-mail: liupin@home.swjtu.edu.cn
}

收稿日期: 2009-09-21; 接受日期: 2010-07-07

中央高校基本科研业务费专项资金 (批准号: SWJTU09BR197, SWJTU09CX076) 和西南交通大学青年教师科研起步基金 (批准 号: 2009Q062) 资助项目

摘要 本文研究了 2-Calabi-Yau 三角范畴上的 2-Calabi-Yau tilted 代数的 almost complete tilting 模. 我们利用 2-Calabi-Yau 三角范畴上的交换关系给出了 2-Calabi-Yau tilted 代数的 almost complete tilting 模有两个 (Bongartz) 补的一系列充分必要条件.

关键词 2-Calabi-Yau 三角范畴 2-Calabi-Yau tilted 代数 tilting 模

MSC (2000) 主题分类 18E30, 16D90

\section{1 引言}

设 $A$ 是代数闭域 $k$ 上的一个有限维代数. 记 $\bmod A$ 为有限生成右 $A$ - 模范畴. 记单 $A$ - 模同构类 的个数为 $n$. 对一个 $A$ - 模 $T$, 我们用 $\operatorname{pd}_{A} T$ 表示它的投射维数, 用 $\operatorname{add} T$ 表示由 $T$ 的所有的直和项 的直和组成的 $\bmod A$ 的满子范畴. 称 $T$ 为 $\bmod A$ 中的 tilting 对象 (或 tilting 模) 如果它满足

1) $\operatorname{pd}_{A} T \leqslant 1$,

2) $\operatorname{Ext}_{A}^{1}(T, T)=0$,

3) 存在正合列 $0 \rightarrow A \rightarrow T^{0} \rightarrow T^{1} \rightarrow 0$, 其中 $T^{0}, T^{1} \in \operatorname{add} T$.

这是 [1] 中 tilting 模的原始定义, 后来 [2] 中证明了上面的第 3) 条可以换成下面的

$3)^{\prime}$ 在同构意义下, $T$ 的不可分解直和项的个数等于 $n$ (有时也称这种情形为模 $T$ 的秩等于代数 $A$ 的秩).

关于 tilting 理论的基本知识请参见 [1-3], 本文中未解释的代数表示论术语请参见 $[4,5]$.

我们称 $A$ - 模 $\bar{T}$ 为 partial tilting 模如果它满足上边定义中的 1) 和 2). 更进一步地, 如果 $\bar{T}$ 在同 构意义下恰有 $n-1$ 个不可分解直和项, 则称 $\bar{T}$ 为一个 almost complete tilting 模. 如果 $A$ - 模 $M$ 满 足 $\bar{T} \oplus M$ 为 $\bmod A$ 中的 tilting 对象并且 $\operatorname{add} \bar{T} \cap \operatorname{add} M=0$, 则称 $M$ 为 $\bar{T}$ 的补. Bongartz 在 [2] 中 证明了对 almost complete tilting 模 $\bar{T}$ 而言, $\bmod A$ 中总存在它的 (Bongartz) 补. 并且由 [6-8] 知 $\bar{T}$ 至 多有两个不同构的补, 确切地说, 在同构意义下, $\bar{T}$ 有唯一的一个补当且仅当 $\bar{T}$ 不是忠实 $A$ - 模, 存在 两个不同的补 $X, X^{\prime}$ 当且仅当 $\bar{T}$ 是忠实的, 此时这两个补由极小逼近序列联系在一起, 即有正合列

$$
0 \rightarrow X^{\prime} \rightarrow B \stackrel{f}{\rightarrow} X \rightarrow 0
$$

其中 $f$ 为极小右 $\operatorname{add} \bar{T}$ - 逼近 ${ }^{[4,5]}$. 
本文的主要目的是研究一般的 2-Calabi-Yau tilted 代数的 almost complete tilting 模的补. 设 $\mathcal{C}$ 为 2-Calabi-Yau 三角范畴, $T$ 为其上的 cluster tilting 对象. $T$ 在 $\mathcal{C}$ 上的自同态代数即为 $T$ 所对应 的 2-Calabi-Yau tilted 代数. 设 $\Gamma$ 为 $T$ 对应的 2-Calabi-Yau tilted 代数, 我们将证明对 $\Gamma$ 而言, 它的 almost complete tilting 模的两个补 (如果有的话) 由 2-Calabi-Yau 三角范畴 $\mathcal{C}$ 上的交换关系联系在一 起, 即给出了 2-Calabi-Yau tilted 代数的交换关系. 这个结果将 Smith 的结果 ${ }^{[9]}$ 从 cluster 范畴推广 到了一般的 2-Calabi-Yau 三角范畴.

\section{2-Calabi-Yau tilted 代数}

设 $\mathcal{C}$ 为 $k$ 上的 $k$ - 线性三角范畴, 这里 $k$ - 线性是指 $\mathcal{C}$ 的 Hom 集都是 $k$ - 向量空间, 态射合成

$$
\operatorname{Hom}(Y, Z) \times \operatorname{Hom}(X, Y) \rightarrow \operatorname{Hom}(X, Z), \quad(g, f) \mapsto g f
$$

对任意 $X, Y, Z \in \mathcal{C}$, 都是 $k$ - 线性的.

设 $\mathcal{C}$ 是幂等可裂的 Krull-Schmidt 范畴, 记 $\mathcal{C}$ 的 suspension 函子为 $S$. 设 $\mathcal{C}$ 为 Hom- 有限的, 即 对任意 $X, Y \in \mathcal{C}$, 态射空间 $\operatorname{Hom}_{\mathcal{C}}(X, Y)$ 都是有限维的.

称 $\mathcal{C}$ 为 2 -Calabi-Yau 三角范畴如果它满足对任意 $X, Y \in \mathcal{C}$, 有

$$
\operatorname{Hom}(X, S Y) \simeq D \operatorname{Hom}(Y, S X)
$$

其中 $D=\operatorname{Hom}_{k}(-, k)$ 为通常对偶.

像通常一样, 对自然数 $n$ 记

$$
\operatorname{Ext}_{\mathcal{C}}^{n}(X, Y)=\operatorname{Hom}_{\mathcal{C}}\left(X, S^{n} Y\right)
$$

2-Calabi-Yau 三角范畴 $\mathcal{C}$ 中的对象 $T$ 称为是 cluster tilting 对象 ${ }^{[10,11]}$, 如果满足

1) $T$ 是 rigid 对象, 即 $\operatorname{Ext}_{\mathcal{C}}^{1}(T, T)=0$;

2) 对任意 $X \in \mathcal{C}$, 如果 $\operatorname{Hom}_{\mathcal{C}}(X, S T)=0$, 则 $X$ 属于 $\operatorname{add} T$.

定理 2.1 ${ }^{[12]}$ 在同构意义下, 2-Calabi-Yau 三角范畴中的 cluster tilting 对象都含有相同个数的 不可分解直和项.

2-Calabi-Yau 三角范畴上的 cluter tilting 对象的自同态代数称为 2-Calabi-Yau tilted 代数.

定理 2.2 ${ }^{[11]}$ 设 $T$ 是 2-Calabi-Yau 三角范畴 $\mathcal{C}$ 上的 cluster tilting 对象, $\Gamma$ 是 $T$ 对应的 2-CalabiYau tilted 代数. 则 $\Gamma$ 的 Gorenstein 维数不超过 1 且

1) 每一个 $\Gamma$ - 模要么是投射 (入射) 维数无限要么是投射 (入射) 维数不超过 1.

2) 2-Calabi-Yau tilted 代数 $\Gamma$ 要么是遗传要么是 global 维数无限.

关于 2-Calabi-Yau 三角范畴和 2-Calabi-Yau tilted 代数, 我们有下面的重要定理来刻画它们之间 的关系:

定理 $2.3^{[11]}$ 设 $T$ 是 2-Calabi-Yau 三角范畴 $\mathcal{C}$ 上的 cluster tilting 对象, 若 $\Gamma$ 是 $T$ 在 $\mathcal{C}$ 上的自 同态代数, 则函子

$$
F=\operatorname{Hom}_{\mathcal{C}}(T,-): \mathcal{C} \rightarrow \bmod \Gamma
$$

诱导出下面的等价:

$$
\mathcal{C} / \operatorname{add} S T \stackrel{\sim}{\rightarrow} \bmod \Gamma
$$


由 [13] 知 2-Calabi-Yau 三角范畴 $\mathcal{C}$ 上有 Auslander-Reiten 变换

$$
\tau_{\mathcal{C}}=S^{2} \circ S^{-1} \simeq S
$$

并且有

命题 2.1 ${ }^{[11]} \mathcal{C} / \operatorname{add} S T \stackrel{\sim}{\rightarrow} \bmod \Gamma$ 上有 Auslander-Reiten 序列, 并且由 $\mathcal{C}$ 上的 Auslander-Reiten 三角诱导.

对 $\mathcal{C}$ 中的态射 $f$, 记 $f$ 为它在投射

$$
\mathcal{C} \rightarrow \mathcal{C} / \operatorname{add} S T \stackrel{\sim}{\longrightarrow} \bmod \Gamma
$$

下在 $\bmod \Gamma$ 中的像. 我们需要下面的定理:

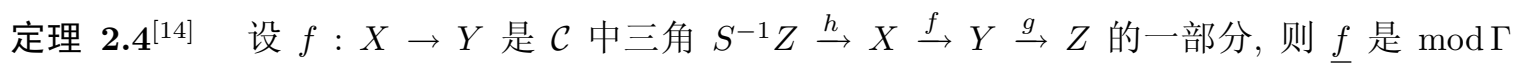
中的单态当且仅当 $\underline{h}=0 ; \underline{f}$ 是 $\bmod \Gamma$ 中的满态当且仅当 $\underline{g}=0$. 进一步, 若 $\underline{S h}=0=\underline{h}$, 则 $0 \rightarrow F X \stackrel{f}{\rightarrow} F Y \stackrel{g}{\rightarrow} F Z \rightarrow 0$ 为 $\bmod \Gamma$ 中的正合列.

\section{3 交换关系}

2-Calabi-Yau tilted 代数的 tilting 模已在 $[9,15,16]$ 中被多位研究者作为主要对象研究过了.下 面的定理对我们来说是非常重要的.

定理 3.1 ${ }^{[15]}$ 设 $T$ 是 2-Calabi-Yau 三角范畴 $\mathcal{C}$ 上的 cluster tilting 对象, $\Gamma$ 是 $T$ 对应的 2-CalabiYau tilted 代数. 若 $L$ 是 $\Gamma$ 的 tilting 模, 则 $L$ 提升到 $\mathcal{C}$ 中的 cluster tilting 对象.

由商范畴的定义, 我们用同一个记号记 $\bmod \Gamma$ 中的模和 $\mathcal{C}$ 中它在投射 $\mathcal{C} \rightarrow \mathcal{C} / \operatorname{add} S T \stackrel{\sim}{\longrightarrow} \bmod \Gamma$ 下的原像.

我们还要用到下面的事实:

定理 3.2 ${ }^{[14]}$ 设 $T$ 是 2-Calabi-Yau 三角范畴 $\mathcal{C}$ 上的 cluster tilting 对象, $\Gamma$ 是 $T$ 对应的 2-CalabiYau tilted 代数. 则 $\mathcal{C}$ 中的 rigid 对象 $L$ 在 $\bmod \Gamma$ 中的像也是 $\operatorname{rigid}$ 的, 亦即若 $\operatorname{Ext}_{\mathcal{C}}^{1}(L, L)=0$, 则 $\operatorname{Ext}_{\Gamma}^{1}(L, L)=0$.

下面我们着重研究 2-Calabi-Yau tilted 代数的交换关系.

设 $T$ 是 2-Calabi-Yau 三角范畴 $\mathcal{C}$ 上的 cluster tilting 对象, $\Gamma$ 是 $T$ 对应的 2-Calabi-Yau tilted 代 数. 若 $\bar{L}$ 为 almost complete tilting $\Gamma$ - 模, $M$ 为它的补, 亦即 $L=\bar{L} \oplus M$ 是 $\bmod \Gamma$ 中的 tilting 模, 其 中 $M$ 为不可分解对象, 则由定理 3.1 知 $L$ 是 $\mathcal{C}$ 中的 cluster tilting 对象. 对此 cluster tilting 对象 $L$, 我们有下面的 2-Calabi-Yau 三角范畴上的交换关系:

定理 $3.3^{[17]}$ 在同构意义下, 存在唯一的不可分解对象 $M^{*}$, 满足 $M^{*}$ 不同构于 $M$, 使得 $L^{\prime}=$ $\bar{L} \oplus M^{*}$ 是 $\mathcal{C}$ 中的 cluster tilting 对象, 并且存在下面的非可裂三角:

$$
M^{*} \stackrel{g}{\rightarrow} B \stackrel{f}{\rightarrow} M \stackrel{h}{\rightarrow} S M^{*} \quad \text { 和 } \quad M \stackrel{f^{*}}{\longrightarrow} B^{\prime} \stackrel{g^{*}}{\longrightarrow} M^{*} \stackrel{h^{*}}{\longrightarrow} S M,
$$

其中 $B$ 和 $B^{\prime}$ 属于 $\operatorname{add} \bar{L}, f$ 是极小右 $\operatorname{add} \bar{L}$ - 逼近, $f^{*}$ 是极小左 $\operatorname{add} \bar{L}$ - 逼近.

定理中的两个三角, 称为 2-Calabi-Yau 三角范畴中的交换三角.

引理 3.1 若 almost complete tilting $\Gamma-$ 模 $\bar{L}$ 是忠实的, 则 $\bar{L} \oplus M^{*}$ 为 tilting $\Gamma$ - 模. 
证明 由 $\bar{L}$ 是忠实的 $\Gamma$ - 模知存在 $M^{\prime} \nsucceq M$ 满足 $\bar{L} \oplus M^{\prime}$ 为 $\bmod \Gamma$ 中的 tilting 对象. 于是由定 理 3.1 知 $\bar{L} \oplus M^{\prime}$ 为 $\mathcal{C}$ 中的 cluster tilting 对象. 再由定理 3.3 和 $M^{\prime} \nsucceq M$ 知, 在同构意义下, $M^{\prime}=M^{*}$, 亦即 $\bar{L} \oplus M^{*}$ 为 tilting $\Gamma$ - 模.

引理 3.2 如定理 3.3 所述, $\bar{L} \oplus M^{*}$ 为 $\mathcal{C}$ 中的 cluster tilting 对象, 若 $M^{*}$ 视为 $\Gamma$ - 模时非零并且 其投射维数不超过 1 , 则 $\bar{L} \oplus M^{*}$ 为 tilting $\Gamma$ - 模.

证明 由 $\bar{L} \oplus M^{*}$ 为 $\mathcal{C}$ 中的 cluster tilting 对象知其为 $\mathcal{C}$ 中的 rigid 对象. 于是由定理 3.2 知 $\operatorname{Ext}_{\Gamma}^{1}\left(\bar{L} \oplus M^{*}, \bar{L} \oplus M^{*}\right)=0$. 由假设, $L^{\prime}=\bar{L} \oplus M^{*}$ 为投射维数不超过 1 的 rigid $\Gamma$ - 模. 于是由 tilting 模的定义知, 要证明 $L^{\prime}$ 为 tilting $\Gamma$ - 模只需证在同构意义下, $L^{\prime}$ 的不可分解直和项的个数与单 $\Gamma$ - 模 的个数一致. 由于 2 -Calabi-Yau 代数 $\Gamma$ 为 cluster tilting 对象 $T$ 在 $\mathcal{C}$ 上的自同态代数, 故在同构意义 下, 单 $\Gamma$ - 模的个数与 $T$ 的不可分解直和项的个数一致. 另一方面, 由定理 2.1 知 cluster tilting 对象 $L^{\prime}$ 与 $T$ 在同构意义下具有相同个数的不可分解直和项个数, 从而引理得证.

下面给出本文的主要结果. 我们提请读者注意, 定理中交换三角的存在性由定理 3.1 和 3.3 保证.

定理 3.4 设 $T$ 是 2-Calabi-Yau 三角范畴 $\mathcal{C}$ 上的 cluster tilting 对象, $\Gamma$ 是 $T$ 对应的 2-Calabi-Yau tilted 代数. 若 $L=\bar{L} \oplus M$ 是 $\bmod \Gamma$ 中的 tilting 模, 其中 $M$ 为不可分解对象, 并且

$$
M^{*} \stackrel{g}{\rightarrow} B \stackrel{f}{\longrightarrow} M \stackrel{h}{\longrightarrow} S M^{*} \quad \text { 和 } \quad M \stackrel{f^{*}}{\longrightarrow} B^{\prime} \stackrel{g^{*}}{\longrightarrow} M^{*} \stackrel{h^{*}}{\longrightarrow} S M
$$

为其对应的交换三角, 则以下各条等价:

(1) $\bar{L} \oplus M^{*}$ 是 tilting $\Gamma$ - 模;

(2) 作为 $\Gamma$ - 模, $M^{*} \neq 0$ 且 $\operatorname{pd}_{\Gamma} M^{*} \leqslant 1$;

(3) 在 $\bmod \Gamma$ 中, $f$ 为满态, 或者 $\underline{f}^{*}$ 为单态;

(4) $\bar{L}$ 为忠实 $\Gamma$ - 模.

证明 显然, 由引理 3.2 知 (1) 和 (2) 等价, 由引理 3.1 知 (1) 和 (4) 等价.

若 (2) 成立, 由引理 3.2 知模 $\bar{L}$ 有两个不同的补 $M, M^{*}$, 且存在下面的正合列 $[6,7]$

$$
0 \rightarrow M^{*} \rightarrow C \stackrel{j}{\rightarrow} M \rightarrow 0,
$$

或

$$
0 \rightarrow M \stackrel{j^{*}}{\rightarrow} C^{*} \rightarrow M^{*} \rightarrow 0
$$

其中 $C, C^{*} \in \operatorname{add} \bar{L}$. 若有正合列

$$
0 \rightarrow M^{*} \rightarrow C \stackrel{j}{\rightarrow} M \rightarrow 0,
$$

并设 $j: C \rightarrow M$ 为 $\mathcal{C}$ 中与 $\underline{j}$ 对应的态射. 由定理 $3.3, f: B \rightarrow M$ 为极小右 $\operatorname{add} \bar{L}$ - 逼近, 故存在 $f^{\prime}: C \rightarrow B$,

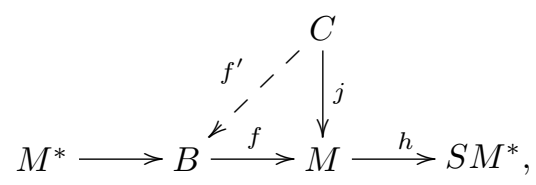

使得

$$
j=f f^{\prime} .
$$

于是 $\underline{j}=\underline{f} \cdot \underline{f^{\prime}}$, 由 $\underline{j}$ 为满态知 $\underline{f}$ 为满态. 同样, 若存在的正合列是

$$
0 \rightarrow M \stackrel{j^{*}}{\rightarrow} C^{*} \rightarrow M^{*} \rightarrow 0
$$


则类似可以证明 $\underline{f}^{*}$ 为单态, 亦即 (3) 成立.

反过来, 若 $\underline{f}$ 为满态, 则由定理 2.4 知 $\underline{h}=0$, 故在 $\mathcal{C}$ 中 $h$ 通过 $\operatorname{add} S T$ 分解, 从而 $S^{-1} h$ 通过 $\operatorname{add} T$ 分解. 又 $\operatorname{pd}_{\Gamma} M \leqslant 1$, 设

$$
0 \rightarrow P_{1} \rightarrow P_{0} \rightarrow M \rightarrow 0
$$

为 $M$ 的投射预解, 由定理 2.2 知 $P_{1}, P_{0}$ 的入射维数不超过 1 , 故 $M$ 的入射维数有限, 从而 $\operatorname{id}_{\Gamma} M \leqslant 1$, 故由 Auslander-Reiten 公式 (参见 $[4,5]$ ) 知

$$
\operatorname{Hom}_{\Gamma}\left(\tau^{-1} M, \Gamma\right)=D \operatorname{Ext}_{\Gamma}^{1}(\Gamma, M)=0 .
$$

由于 $\bmod \Gamma$ 的 Auslander-Reiten 变换由 $\mathcal{C}$ 上的 Auslander-Reiten 变换即 $S$ 诱导 (命题 2.1), 故知在 $\mathcal{C}$ 中从 $S^{-1} M$ 指向 $\operatorname{add} T$ 的态射均通过 $\operatorname{add} S T$ 分解. 又已经知道 $S^{-1} h$ 通过 $\operatorname{add} T$ 分解, 于是我们有 $\underline{S^{-1} h}=0$, 故由定理 2.4 知存在 $\bmod \Gamma$ 中的正合列

$$
0 \rightarrow M^{*} \rightarrow B \rightarrow M \rightarrow 0
$$

由 $\operatorname{pd}_{\Gamma} M \leqslant 1$ 和 $\operatorname{pd}_{\Gamma} B \leqslant 1$ 知 $\operatorname{pd}_{\Gamma} M^{*} \leqslant \infty$, 于是由定理 2.2 知

$$
\operatorname{pd}_{\Gamma} M^{*} \leqslant 1
$$

另外由 $M^{*} \notin \operatorname{add} \bar{L}$ 知 $M^{*} \neq 0$, 即 (2) 成立.

致谢 作者感谢付昌建和彭联刚的有益讨论，并且感谢朱樹和匿名审稿人的非常有价值的建议和评论.

\section{参考文献}

1 Happel D, Ringel C M. Tilted algebras. Trans Amer Math Soc, 1982, 274: 399-443

2 Bongartz K. Tilted algebras. In: Representations of Algebras. Lect Notes in Math 903. Berlin-New York: SpingerVerlag, 1981, 26-38

3 Ringel C M. Some remarks concerning tilting modules and tilted algebras, origin, relevance, future. In: Angeleri-Hügel L, Happel D, Krause H, eds. Handbook of Tilting Theory. London Math Soc Lect Notes Series 332 . Cambridge: Cambridge Univ Press, 2007, appendix

4 Assem I, Simson I, Skowroński I. Elements of the Representation Theory of Associative Algebras, vol. 1: Techniques of Representation Theory. London Math Society Student Texts 65. Cambridge: Cambridge Univ Press, 2006

5 Auslander M, Reiten I, Smalø S. O. Representation Theory of Artin Algebras. Cambridge: Cambridge Univ Press, 1995

6 Happel D, Unger L. Almost complete tilting modules. Proc Amer Math Soc, 1989, 107: 603-610

7 Happel D. Partial tilting modules and recollement. In: Proceedings of the International Conference of Algebra. Contemp Math 131. Providence, RI: Amer Math Soc, 1992, 345-362

8 Riedtmann C, Schofield A. On a simplical complex associated modules. Comment Math Helv, 1991, 66: 70-78

9 Smith D. On tilting modules over cluster-tilted algebras. Illinois J Math, 2008, 52: 1223-1247

10 Iyama O. Higher dimensional Auslander-Reiten theory on maximal orthogonal subcategories. Adv Math, 2007, 210: $22-50$

11 Keller B, Reiten I. Cluster-tilted algebras are Gorenstein and stably Calabi-Yau. Adv Math, 2007, 211: 123-151

12 Dehy R, Keller B. On the combinatorics of rigid objects in 2-Calabi-Yau categories. Int Math Res Not, 2008, 2008: rnn029

13 Reiten I, Van den Bergh M. Noetherian hereditary abelian categories satisfying Serre duality. J Amer Math Soc, 2002, 15: 295-366

14 Koenig S, Zhu B. From triangulated categories to abelian categories: cluster tilting in a general framework. Math Z, 2008, 258: $143-160$ 
15 Fu C, Liu P. Lifting to cluster-tilting objects in 2-Calabi-Yau triangulated categories. Comm Algebra, 2009, 37: 2410-2418

16 Holm T, Jørgensen P. On the relation between cluster and classical tilting. J Pure Appl Algebra, 2010, 214: 1523-1533

17 Iyama O, Yoshino Y. Mutation in triangulated categories and rigid Cohen-Macaulay modules. Invent Math, 2008, 172: $117-168$

\section{Exchange relation for 2-Calabi-Yau tilted algebras}

\section{LIU Pin}

Abstract In this note, we consider 2-Calabi-Yau tilted algebras, i.e., algebras which appear as endomorphism rings of cluster-tilting objects in 2-Calabi-Yau triangulated categories. We show that, given one complement to an almost complete basic tilting module over such an algebra, the other one (if exists) can be constructed using the exchange relation in the corresponding 2-Calabi-Yau triangulated category.

Keywords: 2-Calabi-Yau triangulated category, 2-Calabi-Yau tilted algebra, tilting module $\operatorname{MSC}(2000): \quad 18 \mathrm{E} 30,16 \mathrm{D} 90$ 